\title{
A Chromatographic Method for the Purification of K99 Pili from Enterotoxigenic Escherichia coli
}

\author{
By K. ALTMANN, ${ }^{1}$ N. A. PYLIOTIS, ${ }^{2}$ J. D. WETHERALL ${ }^{3}$ \\ AND T. K. S. MUKKUR ${ }^{4 *}$ \\ ${ }^{1}$ Department of Veterinary Pathology, University of Sydney, Glebe, N.S.W. 2037, Australia \\ ${ }^{2}$ Department of Anatomical Pathology, Prince Henry's Hospital, St. Kilda Road, Melbourne, \\ Victoria 3004, Australia \\ ${ }^{3}$ School of Medical Technology, Western Australian Institute of Technology, Kent Street, Bently, \\ W.A. 6012, Australia \\ ${ }^{4}$ CSIRO, Division of Animal Health, McMaster Laboratory, Private Bag No. 1, Glebe, \\ N.S.W. 2037, Australia
}

(Received 4 June 1982; revised 8 November 1982)

\begin{abstract}
Details of a relatively inexpensive method for the purification of K99 pili in their native conformation are reported. The method involved sequential precipitation of K99 pili with $\left(\mathrm{NH}_{4}\right)_{2} \mathrm{SO}_{4}$, followed by precipitation in $12 \%(\mathrm{w} / \mathrm{v})$ mannose or sorbitol solution. The crude pili preparation was adsorbed with Bio-Gel A-5m and subjected to sequential gel filtration on BioGel A-5m equilibrated with Tris/EDTA/ $/ \mathrm{NaN}_{3} / \mathrm{NaCl}$ buffer and $\mathrm{KSCN} / \mathrm{KCl}$ solution, respectively. The $\mathrm{K} 99$ containing peak was subjected to sequential ion-exchange chromatography on DEAE-Bio-Gel A equilibrated with $0.02 \mathrm{M}$-Tris/ $\mathrm{HCl}, \mathrm{pH} 8.6$ containing $0.05 \mathrm{M}-\mathrm{NaCl}$ and DEAE-Sephadex A-50 equilibrated with 0.05 $\mathrm{m}$-phosphate buffer, $\mathrm{pH} 7 \cdot 2$. The purified pili yielded a single band on SDS-PAGE with an estimated molecular weight of 13000 . Attempts to purify pili by other methods evaluated, viz. $\mathrm{MgCl}_{2}$ precipitation and chromatofocusing were unsuccessful. While the amino acid composition of purified K99 pili was similar to that reported previously the $N$-terminal amino acid was apparently blocked.
\end{abstract}

\section{INTRODUCTION}

A substantial proportion of strains of enterotoxigenic Escherichia coli isolated from field cases of calf and lamb scours, and some of porcine origin have been found to possess pili designated K99 (Guinée et al., 1976; Moon et al., 1976; Myers \& Guinée 1976; Sivaswamy \& Gyles, 1976). Purification of this antigen is necessary for the evaluation of its immunogenic potential in the prevention of colibacillosis. An earlier investigation revealed that K99 pili were composed primarily of two protein subunits, having molecular weights of 22500 and 29500 (Isaacson, 1977). However, recently we found that K99 pili purified from the crude KSCN extracts of enterotoxigenic $E$. coli were different in that (a) they were composed of single subunits, 13000 in molecular weight and (b) while the sulphur-containing amino acids, cysteine and methionine were absent, phenylalanine and tyrosine were present (Altmann et al., 1982). Otherwise the purified pili resembled those described by Isaacson (1977) in sedimentation coefficient, diameter and approximate length (determined ultrastructurally). These results concur, at least partially, with those reported recently by De Graaf et al. (1981) for K99 pili purified by the use of dissociating agents such as urea and sodium deoxycholate. However, the diameter of pili purified in this way was smaller than that reported previously (Isaacson, 1977; Altmann et al., 1982) thus suggesting a loss of their native conformation. The method developed for the purification of K99 pili in this laboratory (Altmann et al., 1982) was expensive in that it involved the use of preparative isoelectric focusing, which requires highly specialized equipment and 
expensive chemicals, viz. pharmalytes. A relatively inexpensive preparative method for obtaining purified K99 pili in their native conformation is described here which should enable the immunogenic properties of purified K99 in experimental colibacillosis to be evaluated.

\section{METHODS}

Bacteria. Enterotoxigenic Escherichia coli (C1443) which was kindly provided by Dr J. Craven of the Veterinary Research Laboratory, 'Attwood' Westmeadows, Victoria, Australia, had the serotype O20:KX106:K99+, $\mathrm{H}^{-}$ (determined by Mr Ian Links, Regional Veterinary Laboratory, Wagga Wagga, N.S.W., Australia) and produced both heat-stable and heat-labile enterotoxins.

Growth conditions. Enterotoxigenic E. coli were grown in Minca medium (Guinée et al., 1976) at $37{ }^{\circ} \mathrm{C}$ in a batch fermenter (New Brunswick) in 101 lots, with aeration at the rate of $31 \mathrm{~min}^{-1}$.

Extraction of K99 pili. Crude preparations of K99 pili were obtained by extraction with $3 \mathrm{M}-\mathrm{KSCN}$ for $30 \mathrm{~min}$ at $22^{\circ} \mathrm{C}$ as described previously (Altmann et al., 1982).

Assays of K99 activity. K99 activity was assayed by double diffusion in $0.85 \%$ (w/v) Agarose C (Pharmacia) using specific K99 antiserum and/or haemagglutination of horse red blood cells using phosphate-buffered saline as a diluent (Morris et al., 1980). The specific activity in precipitating units or hemagglutinating units and the yield of K99 were calculated as described previously (Altmann et al., 1982).

Preparation of antisera. Merino sheep were immunized with crude KSCN extract ( $5 \mathrm{mg}$ protein per animal at each injection) or purified K99 pili ( $2.5 \mathrm{mg}$ protein per animal at each injection) emulsified in incomplete Freund's adjuvant. Four intramuscular injections were given at monthly intervals when the animals were bled and then the sera were stored at $-20^{\circ} \mathrm{C}$.

Assay for heat-labile toxin. The heat-labile enterotoxic activity was assayed using the $y$-1 adrenal tumour cell line (Donta et al., 1974).

Analytical SDS-PAGE. SDS-PAGE was carried out in $10 \%(\mathrm{w} / \mathrm{v})$ acrylamide with standard proteins in the range 68000 to $12100 \mathrm{Dal}$ as described previously (Altmann et al., 1982).

Absorption with Bio-Gel A-5m. Absorption of K99-enriched preparations was carried out with Bio-Gel A-5m equilibrated with TEAN buffer $\left(0.05 \mathrm{M}\right.$-Tris, $0.001 \mathrm{M}-\mathrm{Na}_{2}$ EDTA, $0.003 \mathrm{M}-\mathrm{NaN}_{3}, 0.2 \mathrm{M}-\mathrm{NaCl}$, pH 7.5) containing $10 \%(\mathrm{w} / \mathrm{v})\left(\mathrm{NH}_{4}\right)_{2} \mathrm{SO}_{4}$ (Altmann et al., 1982).

Chromatography. Molecular sieve chromatography was carried out on a column $(1 \cdot 6 \times 70 \mathrm{~cm})$ of Bio-Gel A-5m equilibrated with TEAN buffer (Clements \& Finkelstein, 1979) followed by re-chromatography on the same gel equilibrated with $3 \mathrm{M}-\mathrm{KSCN}$ solution saturated with $\mathrm{KCl}, \mathrm{pH} 8 \cdot 2$ (KSCN/KCl solution). Ion-exchange chromatography was carried out on columns $(1.6 \times 3.5 \mathrm{~cm})$ of DEAE-Bio-Gel A equilibrated with $0.02 \mathrm{M}$ Tris $/ \mathrm{HCl}$ and $0.05 \mathrm{M}-\mathrm{NaCl}, \mathrm{pH} 8.6$ and DEAE-Sephadex A-50 equilibrated with $0.05 \mathrm{M}$-sodium phosphate buffer, $\mathrm{pH} 7 \cdot 2$.

Chemical analysis. Protein concentration was estimated using the Bio-Rad protein kit. The method used for the detection of DNA was that of Ceriotti (1952), RNA was detected by the method of Mukkur \& Pyliotis (1981). The lipopolysaccharide (LPS) content was estimated by using 2-keto-3-deoxyoctonate as a standard as described previously (Mukkur \& Pyliotis, 1981; Altmann et al., 1982). Estimation of total carbohydrates was made using anthrone reagent according to Mokrasch (1954), with glucose as a standard.

Immunoelectrophoresis. Immunoelectrophoresis was carried out using a standard procedure in $0 \cdot 85 \%(\mathrm{w} / \mathrm{v})$ Agarose C. Antisera used for development were sheep anti-K99 and anti-KSCN extracts.

Electron microscopy. Crude and purified K99 preparations were negatively stained in $2 \%(\mathrm{w} / \mathrm{v})$ sodium phosphotungstate and examined in a Philips EM301 electron microscope (Altmann et al., 1982).

Determination of sedimentation coefficient. The $S_{20, w}$ of purified $\mathrm{K} 99\left(1.0 \mathrm{mg} \mathrm{ml}^{-1}\right)$ was determined in a Beckman analytical ultracentrifuge equipped with a multiplex scanner. Centrifugation was carried out at 37020 r.p.m. in a Titanium rotor (An G-Ti) at $20^{\circ} \mathrm{C}$.

Determination of $\mathrm{N}$-terminal amino acid and amino acid composition. The method attempted for the determination of the $N$-terminal amino acid was the dansylation procedure as recommended by Rosmus \& Deyl (1971); ribonuclease was processed in parallel as a control. The amino acid composition of purified K99 pili was determined following hydrolysis with $6 \mathrm{M}-\mathrm{HCl}$ at $110^{\circ} \mathrm{C}$, in vacuo as described previously (Altmann et al., 1982) using a JLC-6AH amino acid analyser.

\section{RESULTS}

\section{Precipitation of $\mathrm{K} 99$ pili with ammonium sulphate}

$\mathrm{KSCN}$-extracted crude $\mathrm{K} 99$ pili were subjected to $\left(\mathrm{NH}_{4}\right)_{2} \mathrm{SO}_{4}$ precipitation $(50 \%$ final saturation) as described earlier (Altmann et al., 1982) with the modification that the precipitate obtained at $14 \%$ saturation was discarded since it did not possess haemagglutinating activity. This procedure resulted in 2.3- to $2 \cdot 5$-fold purification of K99 activity (Table 1 ). While RNA and 
꿍ㅇㅇㅇ용ํำ

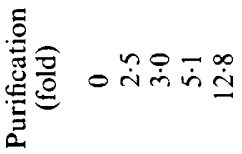$$
\text { 政 }
$$

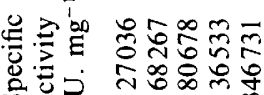

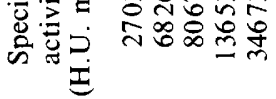
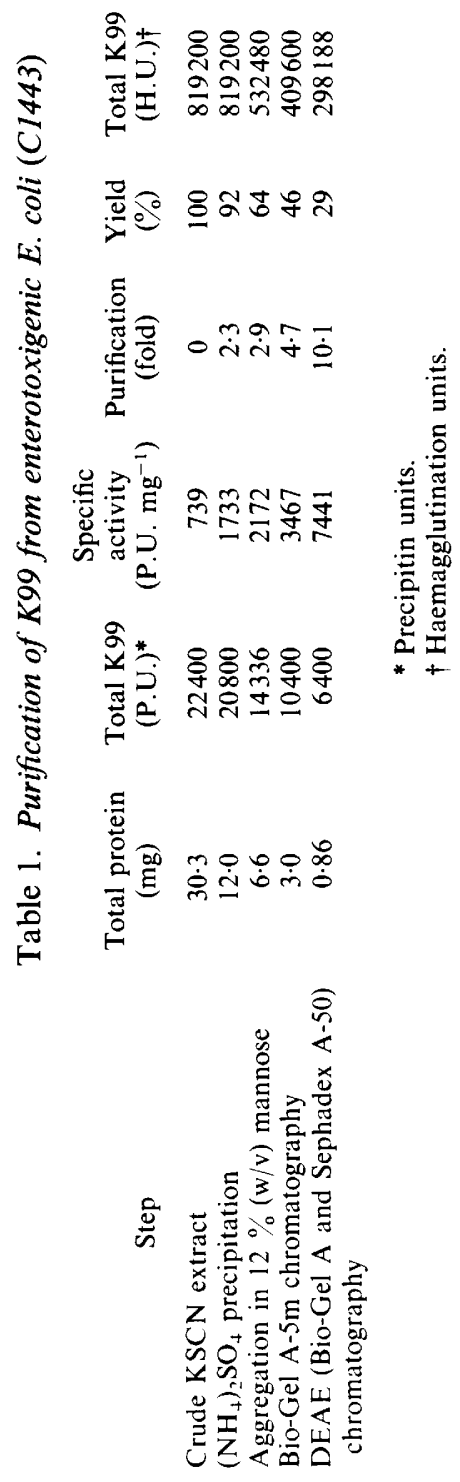


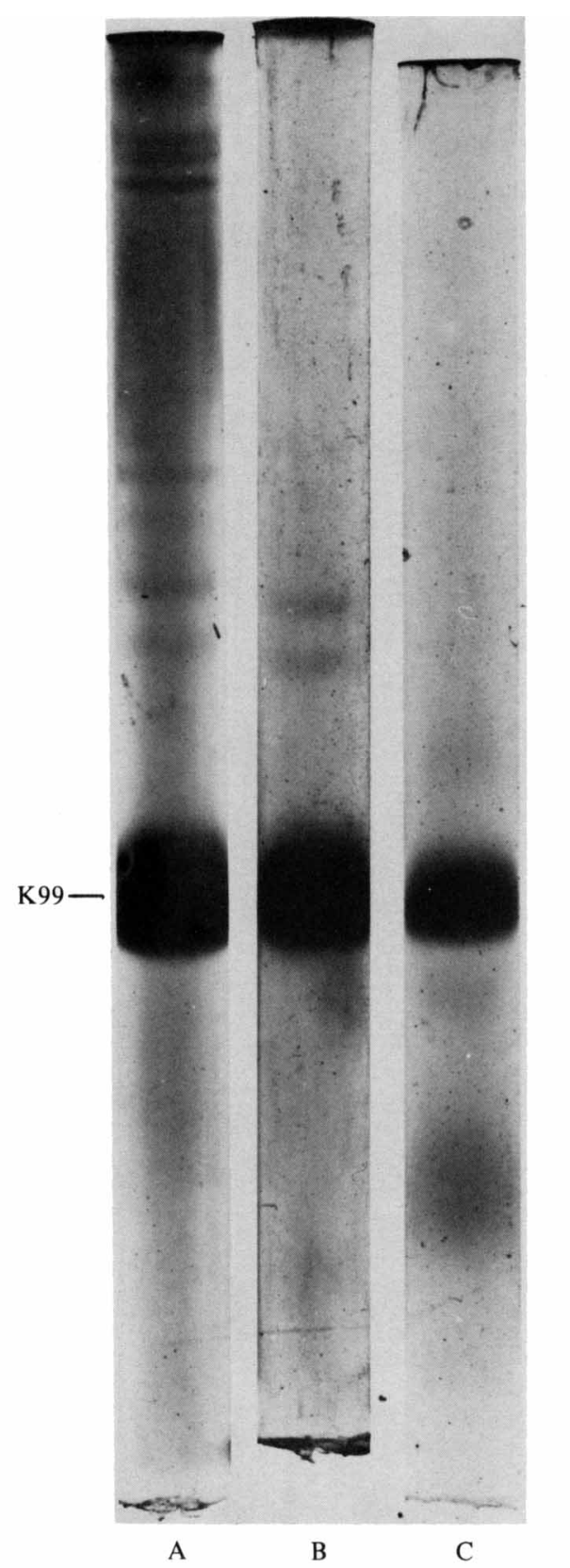

Fig. 1. SDS-PAGE of: A, crude KSCN extract of (100 g) enterotoxigenic Escherichia coli (C1443); B, enriched $\mathrm{K} 99$ obtained following gel filtration on Bio-Gel A-5m equilibrated with $\mathrm{KSCN}-\mathrm{KCl}$ solution $(50 \mu \mathrm{g})$; and $\mathrm{C}$, purified $\mathrm{K} 99(50 \mu \mathrm{g})$.

DNA were still detectable, LPS was absent. On SDS-PAGE, the minimum number of bands staining with Coomassie blue was nine (Fig. 1a).

\section{Precipitation of $\mathrm{K} 99$ pili in $12 \%$ mannose or sorbitol}

The $\left(\mathrm{NH}_{4}\right)_{2} \mathrm{SO}_{4}$ precipitated pili were dissolved in $0.05 \mathrm{M}$-phosphate buffer, $\mathrm{pH} 7.2$ and dialysed extensively against $12 \%(\mathrm{w} / \mathrm{v})$ mannose or sorbitol at $4{ }^{\circ} \mathrm{C}$. The precipitate formed was removed by centrifugation at $27750 \mathrm{~g}$ for $30 \mathrm{~min}$ and dissolved in phosphate buffer. This step 

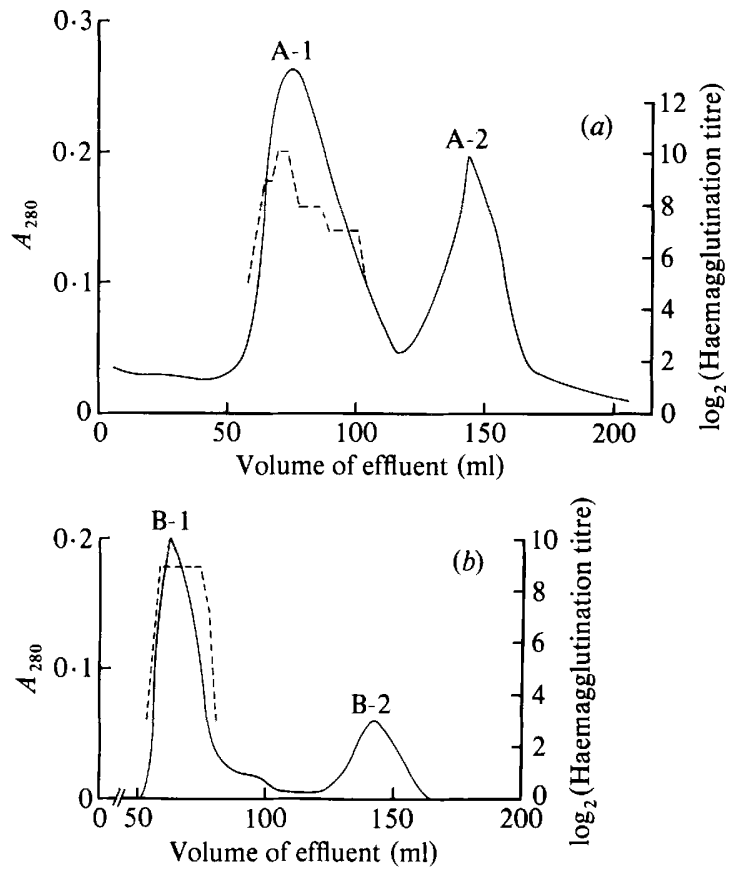

Fig. 2. Elution profiles showing gel filtration of the $\left(\mathrm{NH}_{4}\right)_{2} \mathrm{SO}_{4}$ fractionated pili on Bio-Gel A-5m; (a) column equilibrated with TEAN buffer and $(b)$ re-chromatography of peak A-1 with $\mathrm{KSCN}-\mathrm{KCl}$ solution.

resulted only in a slight increase in purification of K99 (Table 1) but traces of DNA and RNA were removed completely. However, the heat-labile enterotoxic activity was still detectable and no reduction in the number of bands detected by SDS-PAGE was observed.

\section{Molecular sieve chromatography}

Crude pili prepared as above were mixed with Bio-Gel A-5m (Altmann et al., 1982) and the supernatant solution subjected to gel filtration on Bio-Gel A-5m equilibrated with TEAN buffer when two peaks (A-1 and A-2) were eluted (Fig. 2a). K99 activity was confined to peak A-1, the ascending portion of which was pooled and re-chromatographed using the same gel equilibrated with $\mathrm{KSCN} / \mathrm{KCl}$ solution, when two peaks B-1 and B-2 were eluted (Fig. $2 b$ ). Fractions were first dialysed against $0 \cdot 15 \mathrm{M}-\mathrm{KCl}, \mathrm{pH} 8 \cdot 2$, then against phosphate-buffered saline and tested for the presence of K99 activity. The latter was found to be localized to peak B-1 which was pooled and concentrated by pervaporation. No resolution was achieved if phosphate buffer or phosphate buffered saline were used as eluting buffers. The total purification was increased to approximately fivefold (Table 1), although the pili preparation was still heterogeneous as judged by the detection of a minimum of two very faint contaminating bands on SDS-PAGE (Fig. $1 b$ ). Introduction of this step resulted in the removal of the heat-labile enterotoxin as reported previously (Clements \& Finkelstein, 1979; Altmann et al., 1982).

\section{Ion-exchange chromatography}

The preparation obtained by gel filtration on Bio-Gel A-5m was then subjected to ionexchange chromatography on DEAE-Bio-Gel A equilibrated with $0 \cdot 02 \mathrm{M}-\mathrm{Tris} / \mathrm{HCl}, \mathrm{pH} 8 \cdot 6$ containing $0.05 \mathrm{M}-\mathrm{NaCl}$. On elution with the latter buffer, two peaks were eluted (Fig. $3 a$ ) of which only the first peak (peak C-1) possessed $\mathrm{K} 99$ activity. An additional peak (peak C-3) was eluted by $0.02 \mathrm{M}-\mathrm{Tris} / \mathrm{HCl}$, pH 8.6 containing $1.0 \mathrm{M}-\mathrm{NaCl}$ but it contained no $\mathrm{K} 99$ activity. Although peak $\mathrm{C}-1$ contained a single major band on SDS-PAGE, a faint contaminating band with a molecular weight of approximately 40000 could be detected on overloading the gel. This 

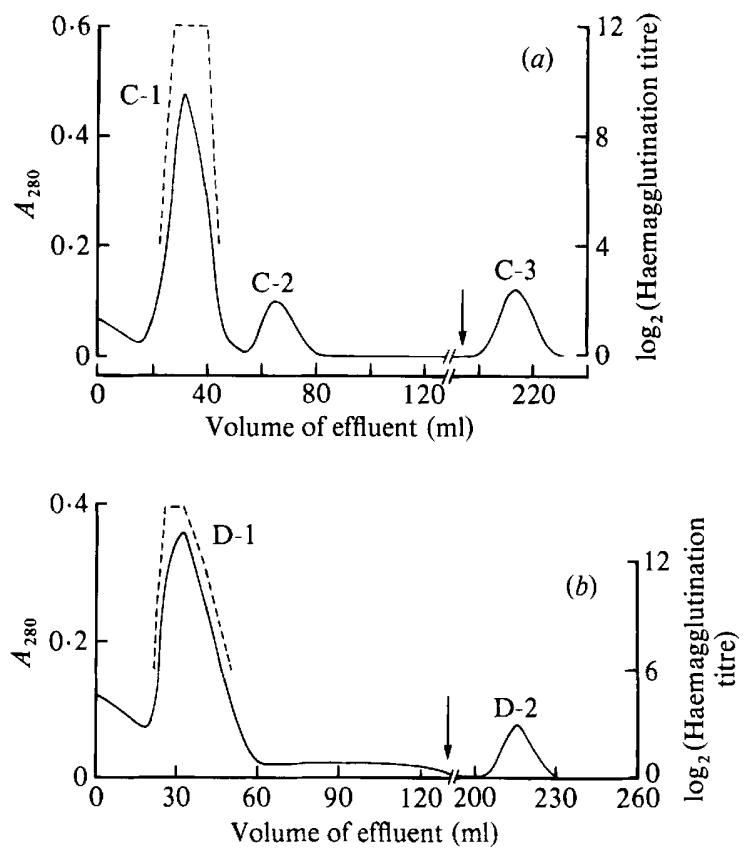

Fig. 3. Discontinuous elution profile showing ion-exchange chromatography of $(a)$ the K99-rich fraction obtained by gel filtration (peak B-1, Fig. $2 b$ ) on DEAE-Bio-Gel A equilibrated with $0.02 \mathrm{M}-$ Tris/ $\mathrm{HCl}$, pH 8.6 containing $0.05 \mathrm{M}-\mathrm{NaCl}$. (b) Peak C-1 eluted in $(a)$ on DEAE-Sephadex A-50 equilibrated with phosphate buffer. The second buffer in both instances consisted of the initial eluting buffer containing $1.0 \mathrm{M}-\mathrm{NaCl}$.

contaminant was removed by chromatography on DEAE-Sephadex A-50, when pure K99 pili were eluted with the equilibrating buffer (Peak D-1; Fig. $3 b$ ).

K99 pili purified by this method yielded a single band on SDS-PAGE with a molecular weight of approximately 13000 (Fig. 1 c). While no DNA, RNA, LPS or heat-labile enterotoxin were detectable in the purified $\mathrm{K} 99$ pili preparation, the amount of carbohydrates measured by the anthrone method was estimated to be $50 \mu \mathrm{g}$ (mg protein $)^{-1}$.

\section{$N$-terminal amino acid analysis and amino acid composition}

The $N$-terminal amino acid was shown to be blocked by the failure to detect an $N$-terminal dansylated amino acid. The amino acid composition of purified $\mathrm{K} 99$ pili was not significantly different from that reported previously (Altmann et al., 1982).

\section{Ultrastructural, immunochemical and biological properties}

As reported previously (Altmann et al., 1982), the average length of purified pili was $160 \mathrm{~nm}$ and the average diameter, $7.4 \mathrm{~nm}$. Further, K99 pili purified by the method described in this investigation fulfilled all the criteria of purity described previously (Altmann et al, 1982). Immunoelectrophoresis of crude KSCN extract yielded a single arc on development with specific anti-K 99 and anti-KSCN extract antisera (Fig. 4) and the $S_{20, w}$ of purified K99 pili was determined to be $12 \cdot 0$.

\section{DISCUSSION}

Recently a new method for the extraction and purification of K99 pili was reported (Altmann et al., 1982). Briefly, the method involved extraction of pili with $3 \mathrm{M}-\mathrm{KSCN}$ followed by $\left(\mathrm{NH}_{4}\right)_{2} \mathrm{SO}_{4}$ precipitation, Bio-Gel A-5m absorption, and preparative isoelectric focusing. This was an effective though expensive method and it was considered appropriate to develop a relatively inexpensive method for the purification of K99 pili. Initial attempts made to purify 


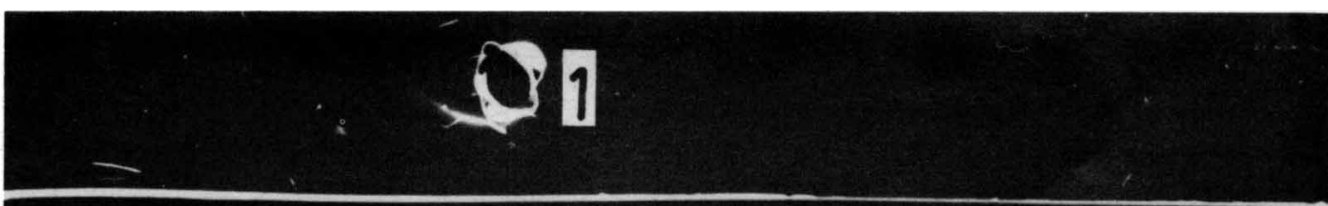

(a)

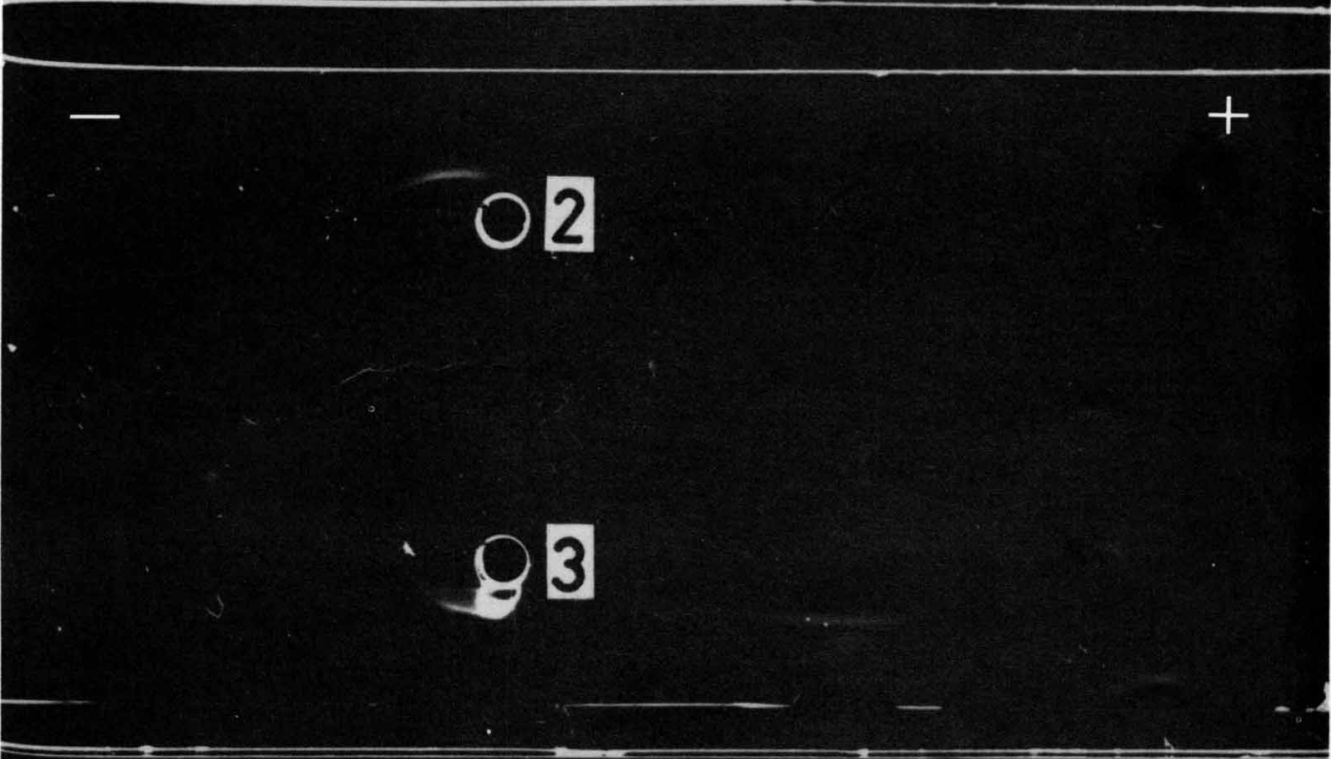

(b)

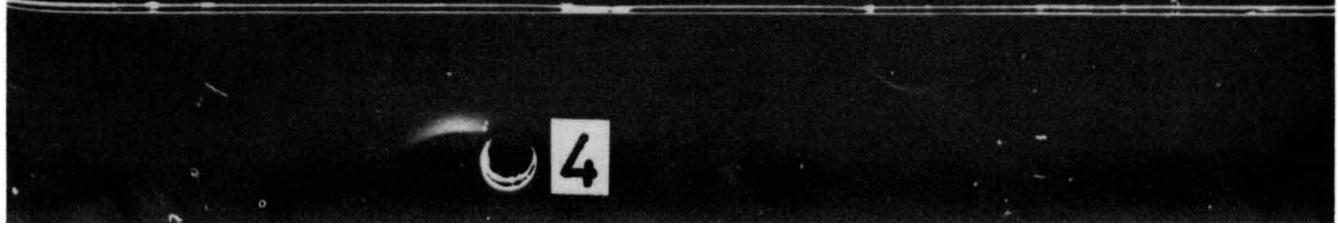

Fig. 4. Immunoelectrophoresis of the crude KSCN extract of enterotoxigenic E. coli (C1443) (wells 2 and 3) and purified K99 (wells 1 and 4). The antisera used for development were (a) antiserum raised against purified $\mathrm{K} 99$ and $(b)$ antiserum against crude $\mathrm{KSCN}$ extract.

K99 pili by the $\mathrm{MgCl}_{2}$ precipitation method of Brinton (1965) were not successful. Since preparative isoelectric focusing had proved effective, the potential of chromatofocusing in achieving the same objective was evaluated. Again, no significant resolution was achieved. Treatment of crude pili preparations with various dissociating agents such as $6 \mathrm{M}$ guanidine. $\mathrm{HCl}, 8 \mathrm{M}$-urea and SDS (Sequenal grade) resulted in significant losses of K 99 activity thus limiting their usefulness. It should be noted, however, that de Graaf et al. (1981) recently reported the isolation of pure pili using urea and sodium deoxycholate. The pili thus purified were $5 \mathrm{~nm}$ in diameter differing from the value $(7.4 \pm 0.6 \mathrm{~nm})$ reported here and by other investigators (Isaacson, 1977; Altmann et al., 1982) and suggesting that the deoxycholatetreated pili did not resume their native conformation. The molecular weight of K 99 pili purified in this investigation was approximately 13000 which, while in agreement with that reported earlier (Altmann et al., 1982), is 5000 to 6000 lower than that recently reported by de Graaf et al. (1981). The possibility that proteolysis occured during the purification of K99 pili described here has not been ruled out. However, the possible existence of K99 antigenic subtypes or the existence of molecular heterogeneity as reported for colonization antigens I and II (Wevers et al., 1980 ) is suggested by the finding that the $N$-terminal amino acid was blocked, whereas de Graaf et al. (1981) found $N$-terminal asparagine. It will be interesting to compare the immunochemical and biological properties of K99 pili purified according to the method of de Graaf et al. (1981) with those prepared according to the method reported here because the quaternary structure of K99 pili reported in this paper has apparently not undergone any gross conformational change. 
In general, the ultrastructural, biological and physicochemical properties of $\mathrm{K} 99$ pili purified in this investigation were similar to those reported earlier (Altmann et al., 1982). Three points that must be emphasized with regard to the method reported here are that (a) the enterotoxigenic $E$. coli must be washed extensively (at least three times) with phosphate-buffered saline until the absorbance of the supernatant obtained upon centrifugation is less than 0.05 at $280 \mathrm{~nm}$, (b) the buffer used for molecular sieve chromatography sequentially on Bio-Gel A-5m must be TEAN (to facilitate removal of heat-labile enterotoxin activity) and $\mathrm{KSCN} / \mathrm{KCl}$ and (c) the samples must be extensively dialysed against appropriate buffers before subjecting them to chromatography. The K99 purified in this investigation apparently represented a homogeneous product because (a) a single band was detected on SDS-PAGE, (b) a single boundary was observed on analytical ultracentrifugation and (c) immunoelectrophoresis of the crude KSCN extract yielded a single arc on development against K99 antiserum prepared by inoculation of sheep with K99 purified by the method described in this investigation. Although the results reported here represent data obtained in a typical experiment using enterotoxigenic $E$. coli C1443, similar results have been repeatedly obtained using the K99 reference strain, B41. It should be possible now to test the potential of purified pili as protective immunogens with a view to understanding their contribution in the pathogenesis of colibacillosis in lambs, calves and piglets.

This project was supported by a research grant made by the Dairying Research Committee of Australia to one of us (T.K.S.M.) at the CSIRO. We extend our sincere thanks to Mrs D. Stewart and Ms S. Tomkins for their technical assistance.

\section{REFERENCES}

Altmann, K., Pyliotis, N. A. \& Mukkur, T. K. S. (1982). A new method for the extraction and purification of K 99 pili from enterotoxigenic Escherichia coli and their characterization. Biochemical Journal 201, 505-513.

Brinton, C. C. (1965). The structure, function, synthesis and genetic control of bacterial pili and a molecular model for DNA and RNA transport in gram-negative bacteria. Transactions of the New York Academy of Sciences 227, 1003-1054.

Ceriotti, G. (1952). A microchemical determination of deoxyribonucleic acid. Journal of Biological Chemistry 198, 297-303.

Clements, J. D. \& Finkelstein, R. A. (1979). Isolation and characterization of homogeneous heat-labile enterotoxins with high specific activity from Escherichia coli cultures. Infection and Immunity 24, 760 769.

DonTa, S. T., Moon, H. W. \& Whipp, S. C. (1974). Detection of heat-labile Escherichia coli enterotoxin with the use of adrenal cells in tissue culture. Science 183, 334-336.

de Graaf, F. K., Klemm, P. \& Gaastra, W. (1981). Purification, characterization and partial covalent structure of Escherichia coli adhesive antigen K99. Infection and Immunity 33, 877-883.

Guinée, P. A. M., Jansen, W. H. \& AgterberG, G. M. (1976). Detection of the K99 antigen by means of agglutination and immunoelectrophoresis in Escherichia coli isolates from calves and its correlation with enterotoxigenicity. Infection and Immunity 13, 1369-1377.

ISAACSON, R. E. (1977). K99 surface antigen of Escherichia coli: purification and partial characterization. Infection and Immunity 15, 272-279.

Mokrasch, L. C. (1954). Analysis of hexose phos- phates and sugar mixtures with the anthrone reagent. Journal of Biological Chemistry 208, 5559 .

Moon, H. W., Whipp, S. C. \& Skartvedt, S. M. (1976). Etiologic diagnosis of diarrheal diseases in calves: frequency and methods for detecting enterotoxin and K99 antigen production by Escherichia coli. American Journal of Veterinary Research 37, 1025-1029.

Morris, J. A., Thorns, C. J. \& SojkA, W. J. (1980). Evidence for two adhesive antigens on the K99 reference strain Escherichia coli B41. Journal of General Microbiology 118, 107-113.

Mukkur, T. K. S. \& Pyliotis, N. A. (1981). The potassium thiocyanate extract of Pasteurella multocida: electron microscopy, and susceptibility of its immunogenic activity to some physical, chemical and enzymatic treatments. Journal of Comparative Pathology 91, 427-437.

Myers, L. L. \& GuinéE, P. A. M. (1976). Occurrence and characteristics of enterotoxigenic Escherichia coli isolated from calves with diarrhea. Infection and Immunity 13, 1117-1119.

Rosmus, J. \& DEYL, Z. (1971). Chromatographic methods in the analysis of protein structure. The methods for identification of $N$-terminal amino acids in peptides and proteins. Part A. Chromatographic Reviews 13, 163-302.

Sivaswamy, G. \& Gyles, C. L. (1976). Characterization of enterotoxigenic bovine Escherichia coli. Canadian Journal of Comparative Medicine 40, 247251 .

Wevers, P., Picken, R., Schmidt, G., Jann, B., JanN, K., Golecki, J. R. \& KIST, M. (1980). Characterization of pili associated with Escherichia coli Ol8ac. Infection and Immunity 29, 685-691. 